\title{
Transient Behaviour of Superconducting Magnet Systems of Fusion Reactor ITER during Safety Discharge
}

\author{
A. M. Miri, ${ }^{1}$ S. Fink, ${ }^{2}$ and W. H. Fietz ${ }^{2}$ \\ ${ }^{1}$ Institute of Electric Energy Systems and High-Voltage Technology (IEH), University of Karlsruhe, Kaiser Street 12, \\ 76128 Karlsruhe, Germany \\ ${ }^{2}$ Forschungszentrum Karlsruhe Institut für Technische Physik, Hermann-von-Helmholtz-Platz 1, \\ 76344 Eggenstein-Leopoldshafen, Germany \\ Correspondence should be addressed to A. M. Miri, miri@ieh.uni-karlsruhe.de
}

Received 29 January 2008; Accepted 6 July 2008

Recommended by Igor Kotenko

To investigate the transient behaviour of the toroidal and poloidal field coils magnet systems of the International Thermonuclear Experimental Reactor during safety discharge, network models with lumped elements are established. Frequency-dependant values of the network elements, that is, inductances and resistances are calculated with the finite element method. That way, overvoltages can be determined. According to these overvoltages, the insulation coordination of coils has to be selected.

Copyright ( 2008 A. M. Miri et al. This is an open access article distributed under the Creative Commons Attribution License, which permits unrestricted use, distribution, and reproduction in any medium, provided the original work is properly cited.

\section{Introduction}

The International Thermonuclear Experimental Reactor (ITER) is the step between today's studies of plasma physics and tomorrow's production of electricity by fusion power plants [1]. The plasma is confined by the magnetic fields of the toroidal field (TF) coils, the poloidal field (PF) coils, and the central solenoid (see Figure 1).

Due to the required huge magnetic fields, the coils need to be superconducting. In the event of a quench, when the conductor changes from superconducting to normal conducting state, the stored magnetic energy must be discharged from the coil by a fast discharge circuit. Thus damage through overheating is avoided. To initiate the fast discharge, counteracting current switches are used which can cause high-voltage transients across the winding. These transients can lead to internal oscillations with overvoltages.

In this investigation, the transient behaviours of the ITER toroidal field (TF) and poloidal field (PF) coils are simulated during safety discharge.

The TF coils are equal and in series. The TF coil system consists of 18 TF coils and 9 fast discharge units (FDUs). The structure of the system is shown in Figure 2. After the determination of the transient behaviour of the system in Figure 7 , the resulted voltage across one TF coil terminal will be applied for further investigations of the detailed model of one coil.

The PF coils have different diameters, winding numbers, and structures. The PF coils 2, 3, 4, and 5 are connected in parallel and are excited according to Figure 3. PF coils 1 and 6 are excited separately. Because of their geometry, for PF3 and PF6 the lowest resonance frequency is assumed. So the models of these two coils are established to determine the insulation stress inside the windings of the PF coils during safety discharge.

For PF coil 3, the lowest resonance frequency was determined. So in safety discharge, only the electrical circuit of PF2 to PF5 is considered. All coils and converters are in parallel. The main purpose of the vertical converter is to control the vertical plasma displacements. To verify the calculation methods, some measurements were performed with a single TF model coil, which has a reduced size compared to the future TF coils [2-6].

\section{The ITER TF and PF Coils and Their Network Models}

The TF coils are "D" shaped and consist of 192 windings. The winding pack is enclosed in a steel case which consists of 


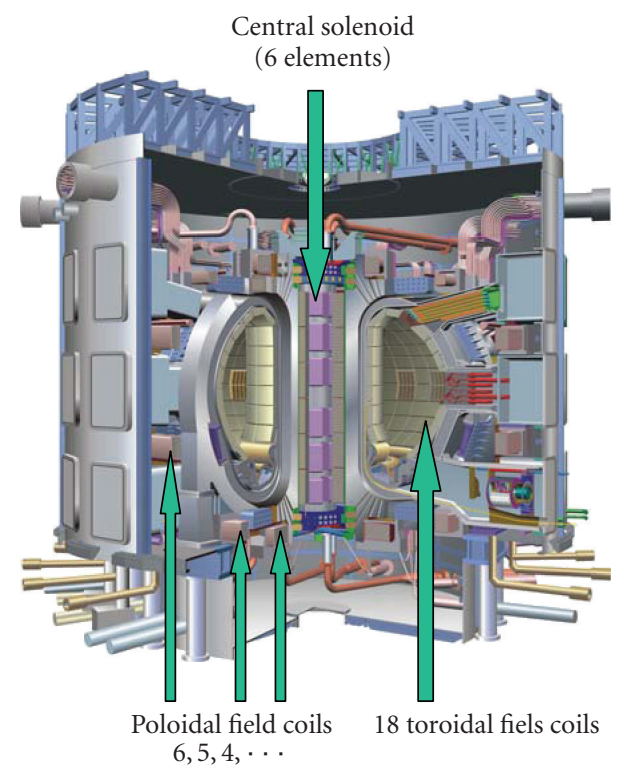

Figure 1: Sectional view of ITER tokamak-reactor.

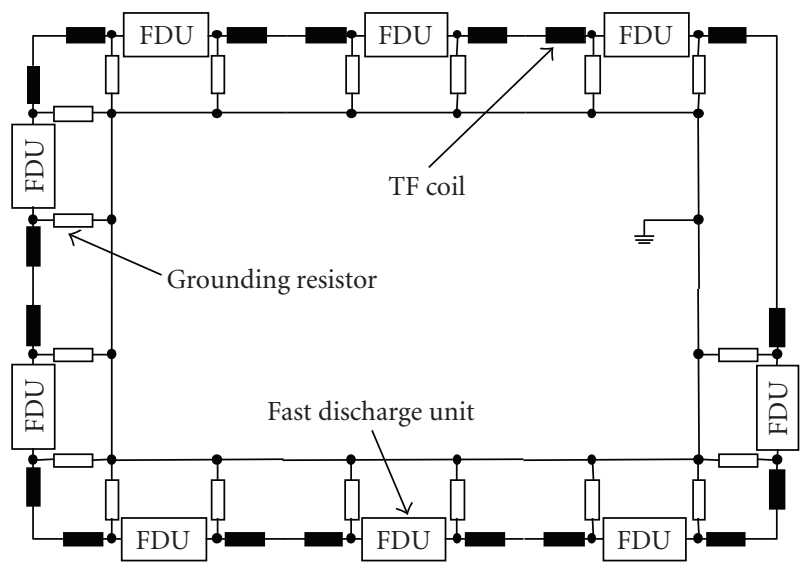

FIGURE 2: Simplified analogue circuit of the TF coil system during a fast discharge (power supply already disconnected).

7 radial plates (see Figure 4). The conductors are surrounded by an insulation made of epoxy resin impregnated kapton and glass [7]. The coil terminals project out of the case on the lower curved part of the case. All other double pancake joints (i.e., joints linking radial plates) are contained within a local extension of the case.

The PF coils are in ring form and have different number of turns. The structure is given in Figure 5.

The 6 PF coils are placed circular around the outboard legs of the TF coils. The PF coils are self-supporting as regards the radial magnetic loads and the vertical loads transmitted to the TF coil cases by supports designed to allow a free radial expansion of the coils. The lead of the PF coil is made up of square steel conduit, which contains the circular superconductor. The superconducting material is the alloy niobium-titanium. It is included as multiple filaments strands in the circular conductor. The square conduit is surrounded by insulation made of epoxy resin-impregnated polyimide film and dry glass.

\subsection{TF Network Model}

Each TF coil consists of 134 turns embedded in radial plates (see Figure 4). Each turn has been modelled by a resistance, a capacitance, and an inductance. The 7 double pancakes are connected in series. The capacitance represents insulation between the turn and the radial plate. The radial plates have capacitive coupling between each other and to the grounded case. From [8], it is proved that the radial plates connected with the centre of double pancake (location of helium inlet) through a resistance is the best linking method. For ITER TF, the value of the resistance is given as $2 * 50 \mathrm{k} \Omega=100 \mathrm{k} \Omega$. Additionally, the influences of instrumentation cables have been considered here. The instrumentation cables are represented with additional capacitances between helium inlet and ground. Figure 7 shows the built network model for one TF coil. The frequency-dependant damping of the radial plates is treated by an FEM model.

\subsubsection{Frequency Sensitivity of Resistances}

Measurements have shown that the superconducting filaments do not participate in high-frequency oscillations for fast transient processes in cable-in-conduit conductors (CICCs) of the type similar to the ones used in the ITER TF $[3,4]$. A possible reason is that at $4 \mathrm{~K}$, the skin depth in the copper matrix surrounding the filaments is very small already for frequencies in the range of $\mathrm{kHz}$. Furthermore, the flux penetration of the superconductor is also related to a time constant. In other words, the transient wave propagates in the surrounding copper matrix. Hence, for the network model, the superconducting strands were regarded conducting in a normal way.

For the total DC resistance of the coil, a value of $1.0 \mathrm{~m} \Omega$ was found, assuming a residual resistance ratio (RRR) [9] of the stabilizing copper of 120 . It can be proved that there is no effective skin or proximity effect for the conductor as a whole. A frequency dependence of the resistance is only effective due to a local current displacement inside the strands by a local skin effect and due to a local proximity effect among the strands. To calculate the frequency-dependent resistance $R_{\text {_skin }}(f)$, an analytic formula or a finite element method (FEM) can be used. The FEM (it refers to the programme Maxwell [9] in this project) has been chosen to do the calculation of the ratio of resistance by certain frequencies to DC values. Some results are shown in Table 1.

\subsubsection{Turn-to-Radial Plate Capacitance}

The capacitance between turns and radial plates can be regarded as cylindrical capacitors [2]. The capacitance between one turn of layer $k$ and the radial plate is given by

$$
C_{k, T \text { to } R}=2 \pi \varepsilon_{0} \varepsilon_{r} \frac{l_{k}}{\ln \left(R_{a} / R_{i}\right)},
$$




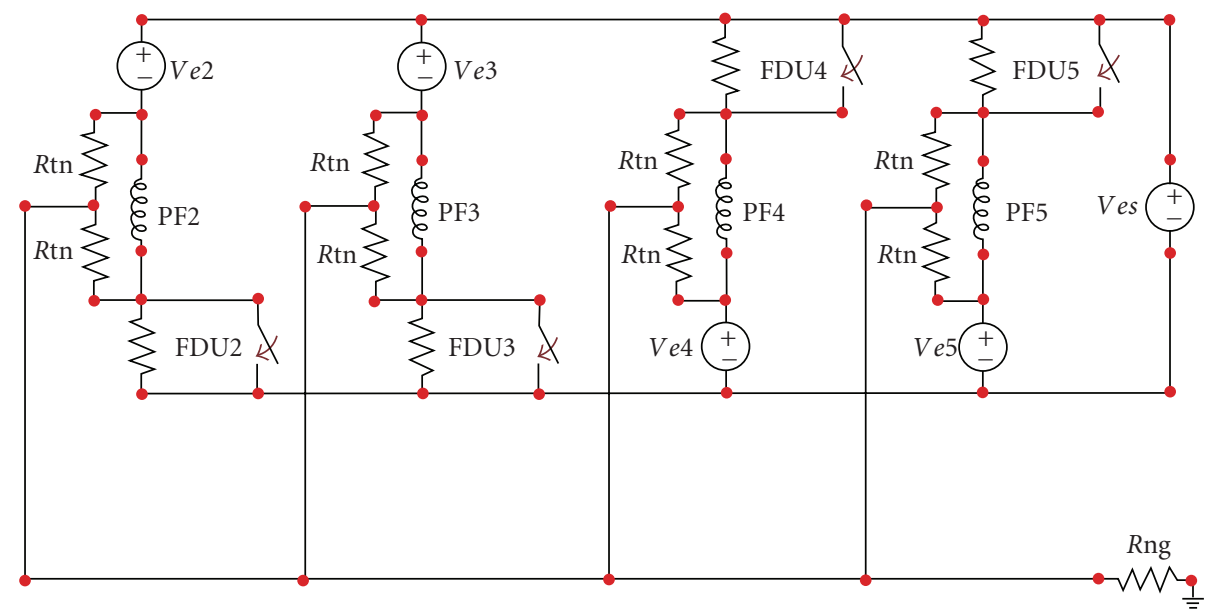

FIgure 3: Outer PF coil electrical circuit PF2, PF3, PF4, and PF5.

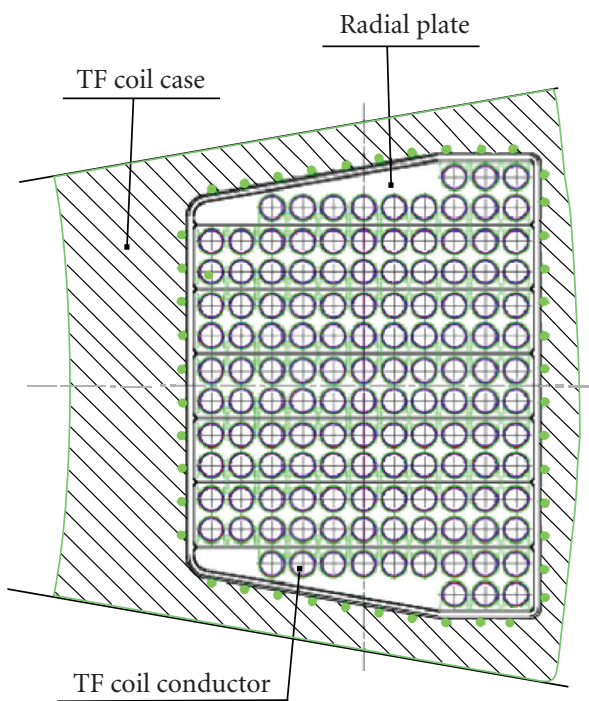

FIgURE 4: Cross-section of one TF coil.

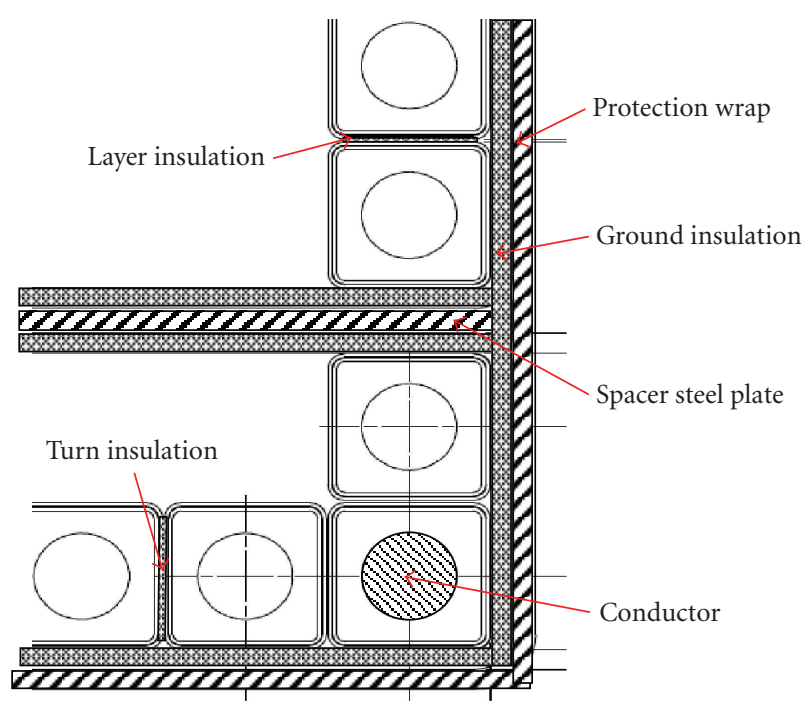

FIGURe 5: Part of the cross-section of an ITER PF coil. where $R_{a}$ and $R_{i}$ are the outer and inner radii of the winding. $l_{k}$ is the length of the winding of layer $k(k=1, \ldots, 11)$.

\subsubsection{Radial Plate to Radial Plate Capacitance}

The capacitance between neighbouring radial plates can be regarded as plate capacitors. The capacitor between two radial plates is given by

$$
C_{R \text { to } R}=\varepsilon_{0} \varepsilon_{r} \frac{L * W}{d},
$$

where $L=34.1 \mathrm{~m}(8$.) is the length of the coil centre line. $W$ is the width of the radial plate in the direction from layer 1 to layer 11 and $d$ is the insulation thickness between the radial plates. It can be proved that this formula has a good approximation compared with the formula with the application of different radii.

\subsubsection{Ground Capacitance}

There are considerable ground capacitance at the innermost and the outermost layer. For the calculation of the capacitances, (2) applies.

\subsubsection{Capacitance of Instrumentation Cables}

The instrumentation cables of $60 \mathrm{~m}$ length cause considerable ground capacitances. It is assumed that the capacitance per unit length is $0.4 \mathrm{nF} / \mathrm{m}$.

\subsubsection{Self- and Mutual Inductances}

The self- and mutual inductances of DC value are to be calculated using analytical formulae for circular cylindrical conductors [10]. The self-inductance of one turn of layer $k$ is given by

$$
L_{k}=\frac{\mu_{0}}{2 \pi} * l_{k} *\left(\ln \left(\frac{4 l_{k}}{d}\right)-0.75\right),
$$


TABLE 1: Resistance ratio.

\begin{tabular}{lc}
\hline Resistance ratio & Frequency $(\mathrm{Hz})$ \\
\hline 25 & 100 \\
37 & 170 \\
108 & 470 \\
533 & 20000 \\
860 & 50000 \\
\hline
\end{tabular}

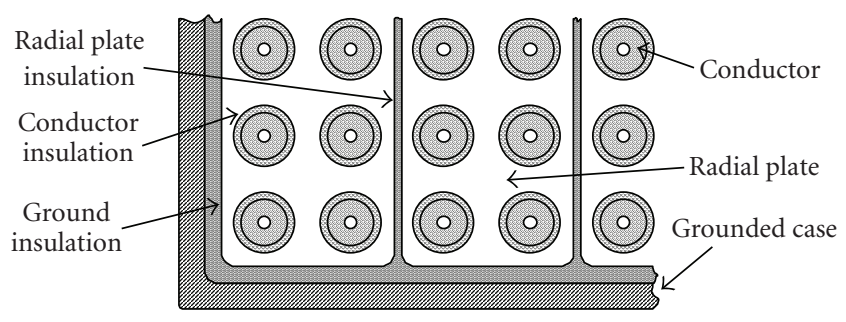

FIgure 6: Part of the cross-section of an ITER TF coil showing the three different types of the electrical insulation.

where $l_{k}$ is the length of layer $k$ and $d$ is the diameter of the winding.

The mutual inductance between $k$ th winding and $j$ th winding is calculated by using the Neumann equation

$$
M=\frac{\mu_{0}}{4 \pi} * \frac{\oint_{l 1} \oint_{l 2} d l_{1} d l_{2}}{r_{12}},
$$

where $l_{1}$ is the length of the $k$ th winding and $l_{2}$ is the length of the $j$ th winding. $d l_{1}$ is the length element of the $k$ th winding, $d l_{2}$ is the length element of the $j$ th winding, and $r_{12}$ is the distance between the two windings.

The total inductance of one TF coil was calculated in program MATLAB with the value of $0.3498 \mathrm{H}$, which agrees well with the value of $0.349 \mathrm{H}$ found in literature [5]. The calculations for the DC values of self- and mutual inductance are discussed above.

During the transient process, there are many different frequency portions, which are included in a voltage signal or a current signal. To get the right frequency response, it is necessary to set the specific inductance values in the model for the corresponding frequencies. That means the frequency-dependent inductances need to be calculated. This has been done with the use of the finite element method (FEM), because the eddy current effect cannot be described through analytical formula.

The FEM discretises 2D or 3D areas. Areas with complex structures are divided into small triangles, so that the interior of a triangle can approximately be handled as homogeneous. A continuum is idealised with a finite number of elements that are connected with nodes. This solution converges towards the exact solution. More detailed information can be read in [11].

\subsubsection{Confirmation of the First Dominant Resonant Frequency}

To make a better statement about the frequency response to the stimulating signals, which contain different frequencies, it is necessary to know the first dominant resonant frequency or the so-called resonance frequency of the single coil network model. Due to the skin effect, at high frequencies, the current is displaced from the inner area of the conductor. So there is a frequency dependence: the $\mathrm{AC}$ resistance of the conductor increases and the inductance decreases.

So the real resonance frequency of the coil cannot be calculated directly. Application of iterative method is necessary: with the FEM-using programme Maxwell, the DC values for the resistances and inductances are calculated. Then the values for the lumped elements are inserted in the transient program ATP [12] and the resonance frequency is determined. With this new resonance frequency, the FEM is applied again. This procedure is carried out iteratively until the resonance frequency converges to the real resonance frequency of the coil. At last $50 \mathrm{kHz}$ resulted in this calculation for a single ITER TF coil.

\subsubsection{Simplified Network Model of ITER TF Coil System without and with Earth Capacitances}

For the magnets, one of the important parameters is overvoltage on the coil terminals that can cause an insulation breakdown and can trigger a chain of other fault events. To get the voltage across the coil terminals and for the transient analysis in the fast discharge unit (FDU), the circuit for TF coil system analysis [16] is used (see Figure 8 ). The TF coil here has been simplified as a pure inductance. This is true when the event of discharge is only analysed without any further fault conditions. But if there is a short circuit on the coil terminals to ground, the capacitances between the coil terminals and ground must be considered for the analysis of the voltage across the coil terminals and other parameters in the FDUs [8].

A replacement of the 18 pure inductances in Figure 8 by 18 coupled single coil models like in Figure 7 would cause huge calculation time. Therefore, capacitances are summarised and distributed within the circuit. The network model of the complete system with the simplified coils is shown in Figure 9. In both cases, the coupling factors among all the $18 \mathrm{TF}$ coils were considered. The values for the coupling factors were taken from [5].

In Figure 9, the earth capacitances are divided into two parts. One part represents the resulted insulation between the radial plates and ground; additionally the resistance with the value of $1 \Omega$ formed by the case has been taken into account as well. Another part represents the capacitances formed by the instrumentation cables, hence the inductance of $\mu \mathrm{H}$ from the instrumentation cable has also been considered. 


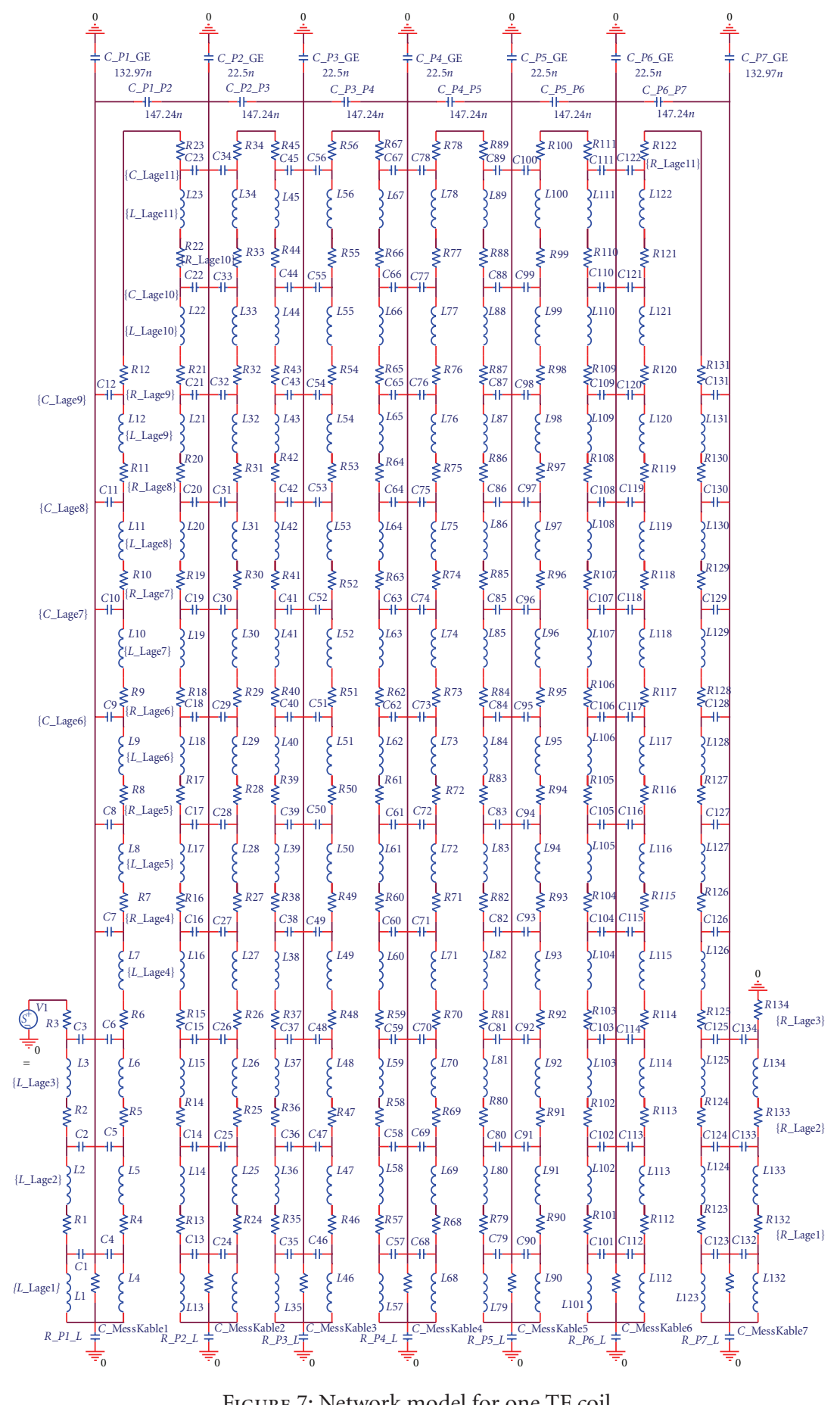

FIgURE 7: Network model for one TF coil.

\subsubsection{The Admissibility of the Network Model}

The admissibility of the network model can be evaluated by calculating the frequency range. The propagation velocity is given by

$$
c=\frac{c_{0}}{\sqrt{\mu_{r} \varepsilon_{r}}}=1.5 * 10^{8} \mathrm{~ms} .
$$

\subsection{PF Network Model}

The maximum permitted frequency for the coil is

$$
f_{\max }=\frac{c}{8 l}=1.1 \mathrm{MHz} \quad(\text { with } l=34.1 \mathrm{~m}) .
$$

As the resonance frequency is much lower, the network model is admissible. 


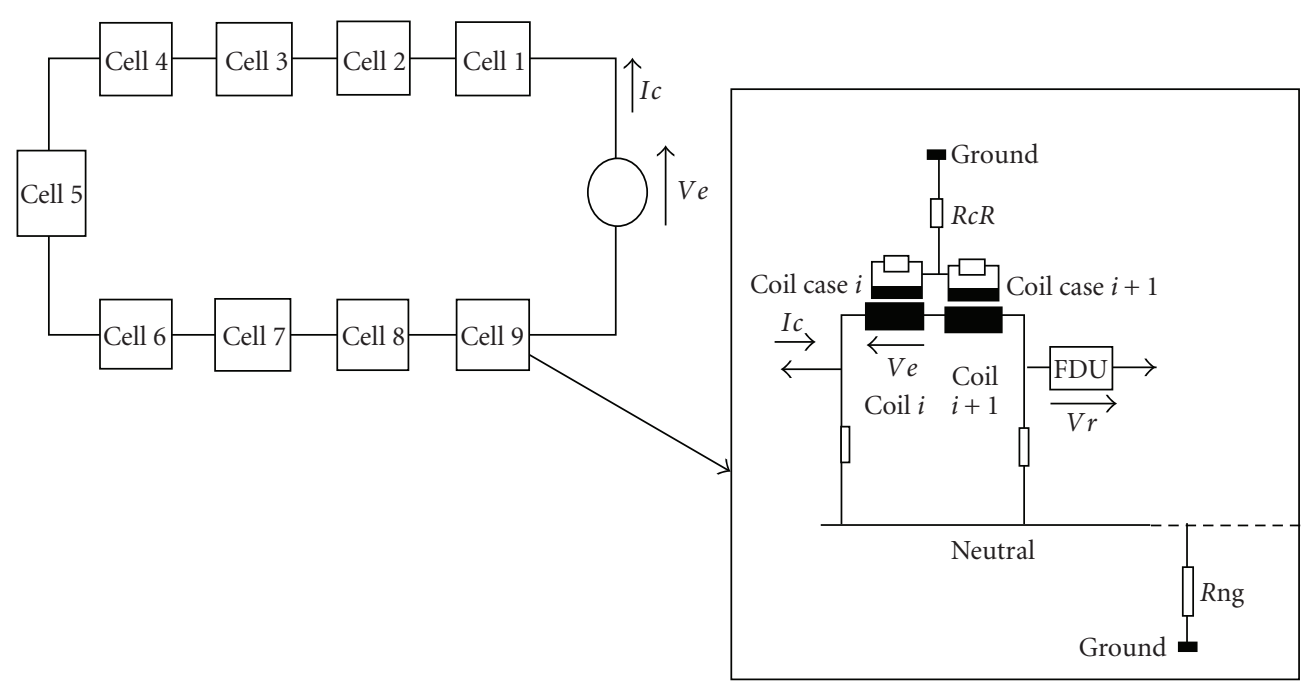

FIGURE 8: Circuit for TF coil system analysis.
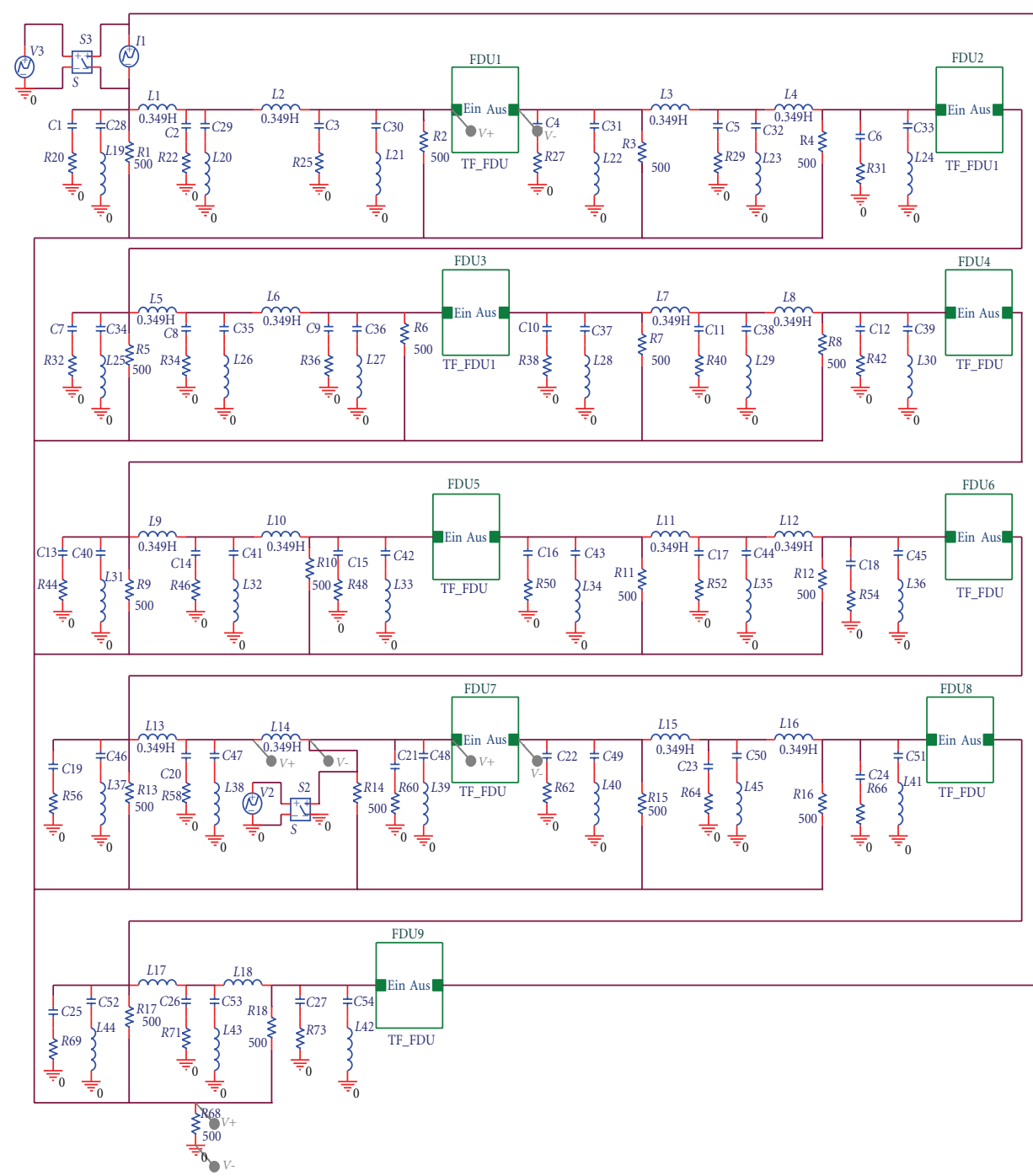

FIGURE 9: Network model of the ITER TF coil system, the 9 FDUs are shown as blocks. 

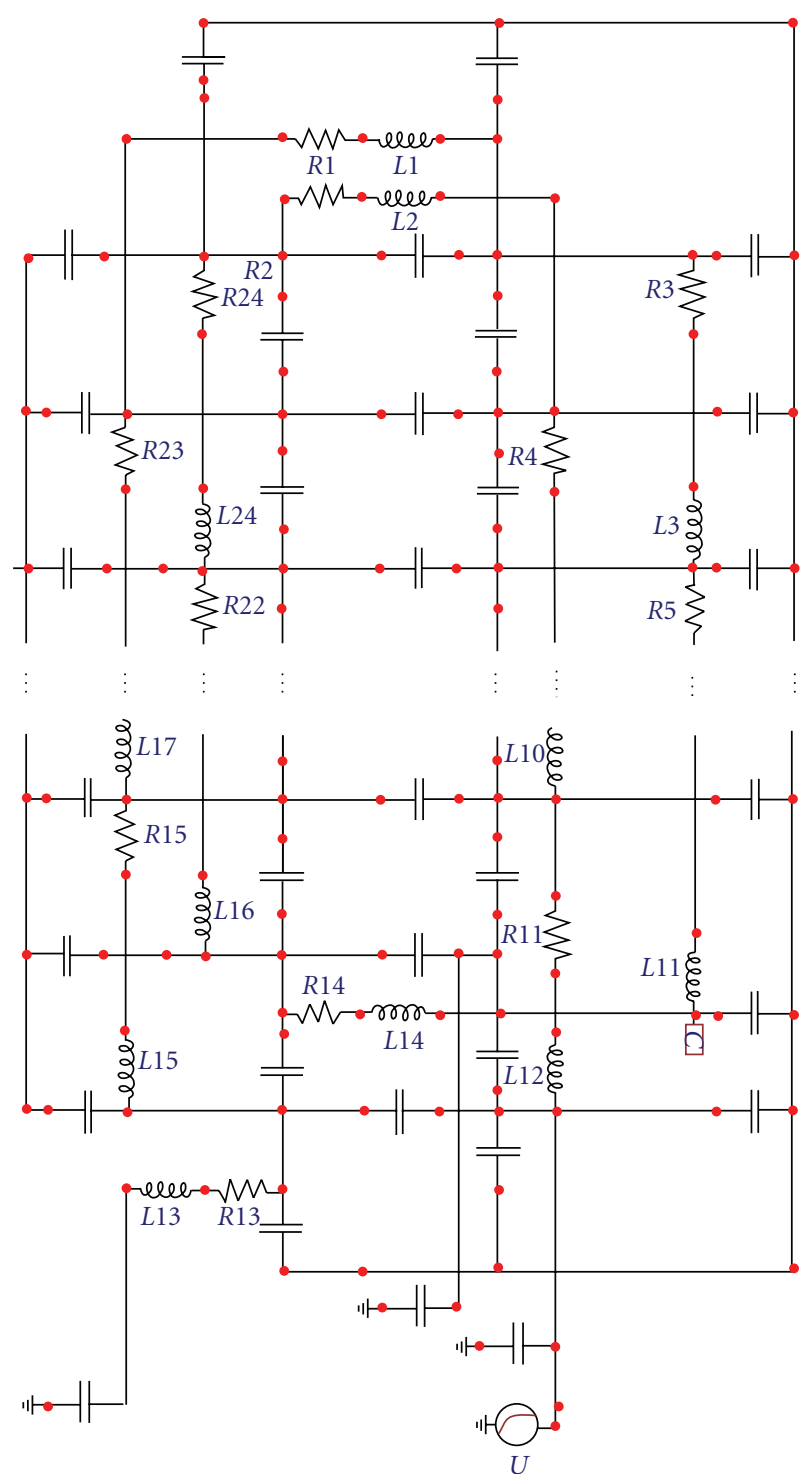

Figure 10: Network model of one double pancake of PF coil 3.

For the establishment of the models for PF coil 2 and coil 3, the procedure is the same as for the TF coils. In Figure 10, the network of PF3 is shown.

\section{Safety Discharge by TF and PF Coils in Normal and in Fault Cases}

If a quench (i.e., the conductor is no longer superconducting) is detected, the fast discharge is initiated to extract the stored energy of the coils and to avoid damage.

The current is commutated from the continuous bypaths to the vacuum circuit breaker paths (see Figure 11) and the power supply is short circuited and switched off. The switches connect the charged counter pulse capacitors. The counter current runs through the vacuum circuit breaker (VCB) in the opposite direction to the coil current. The resulting current decreases.
After this current reaches a zero-crossing and the arc in the circuit breaker extinguishes, the circuit breaker opens. The coil current commutates from the short current path to the damp resistor path where the energy is abolished in the damping resistor $\left(R_{\mathrm{d}}\right)$.

The maximum voltage across the damp resistor of the TF system is in the case of $60 \mathrm{KA}$ about $6 \mathrm{kV}$ (see Figure 12). As at the moment of commutation, the counter pulse capacitor is not completely discharged, the discharge continues over the snubber circuit in the snubber resistance $\left(R_{\mathrm{sn}}\right)$, resulting in a $2 \mathrm{kV}$ pulse at the FDU terminal with a rise time of about 1 microsecond. The currents are shown in Figure 13.

The transient behaviour of the coils is determined for two different cases.

\subsection{Normal Case}

A quench is detected and the energy stored in the coil must be extracted. The TF coil system is excited with a current of $68 \mathrm{kA}$. When the current is switched off, the safety discharge is carried out.

The maximum voltage across the TF coil terminals is investigated with the model in Figure 2. The detailed model like in Figure 9 of a single TF coil is excited with this voltage and the maximum voltage in this coil is determined [6].

For the PF coils, as excitation, a step function with a rise time of 1 millisecond from $1 \mathrm{kV}$ to $9 \mathrm{kV}$ to the network (see Figure 10) is applied.

\subsection{Fault Case}

The single TF coil models $(1 \mathrm{kHz}, 50 \mathrm{kHz})$ are examined for fast discharge (see Figure 14). Maximum voltages are determined for normal case and two fault cases on every type of insulation. The results are summarised in Table 2. For all cases, the first 5 seconds are used for charging and running a DC current of $68 \mathrm{kA}$ through the coils.

The malfunction of the two FDUs was examined in fault case 1 , that is, the commutation in the resistor path is only successful for seven FDUs while the current remains in the bypass path for two FDUs. The voltages from different terminals to ground can be seen in Figure 15.

For the second fault case, it was assumed that the voltage imbalance in the system, caused by fault 1, with the increase of terminal to earth voltages, leads to an earth fault at the time and location of the maximum voltage to earth. For this second fault case, the rise times are in the range of few microseconds for the maximum radial plate and conductor insulation voltages, and in the range of some milliseconds for the maximum ground insulation voltage. The reason for such a difference among the rise times is that the maximum terminal voltage to ground and the maximum voltage between the terminals of a coil were not found at the same coil in Table 3 [14]. The maximum voltages are shown in Figure 16.

The TF coil system was analysed with PSpice programme package where the coupling matrix could be included. Calculations lead to the result, that it can be disregarded for increasing frequency, especially resonance frequency. 


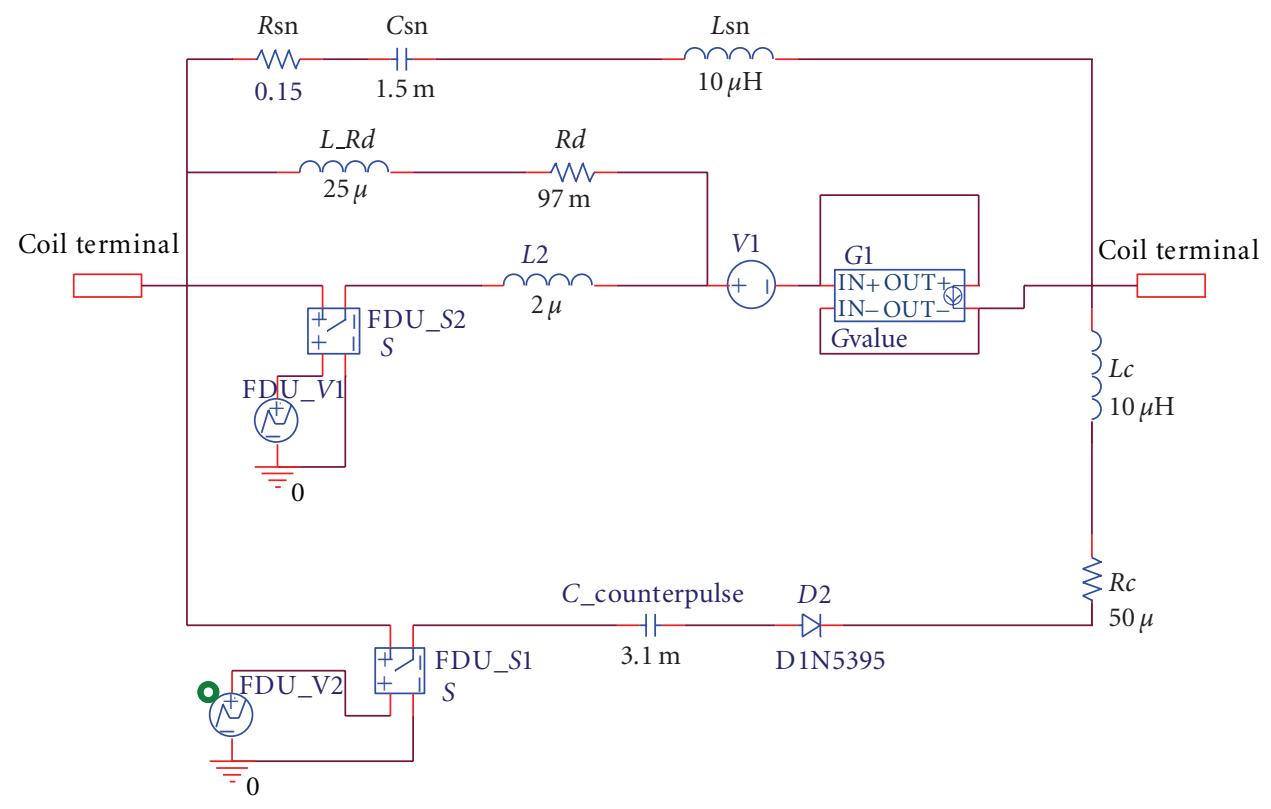

FIGURE 11: Model of the fast discharge circuit of the TF coil.

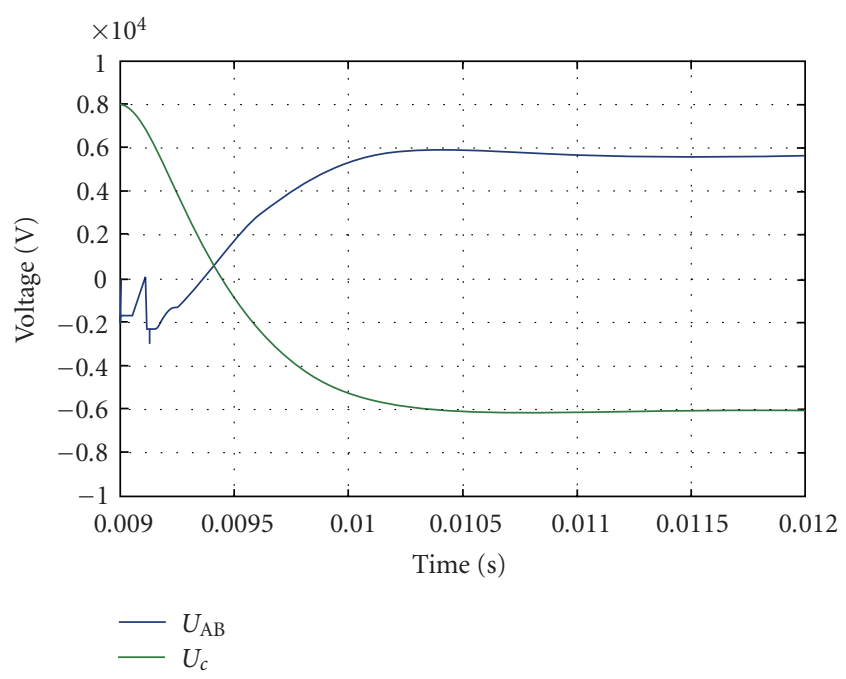

FIgURE 12: Results of TF fast discharge transient analysis; $U_{\mathrm{AB}}$ : voltage across the FDU; $U_{c}$ : voltage on the capacitor; the above results have a good agreement compared with [10].

To determine the PF coil system, similar analyses are performed. During fast discharge in a circuit as shown in Figure 3, all possible combinations of malfunction of one and two FDUs are simulated. The maximum potential to ground of $4.6 \mathrm{kV}$ occurs at the upper terminal of PF coil 4 with FDU5 not opening.

So for the PF coils, fault case 1 means the malfunction of only one FDU, fault case 2 is fault 1 with an earth fault. This earth fault occurs at the terminal where the potential maximum appears after switching off the power supply. The detailed coil model of PF coil 4 is similar to the model of $\mathrm{PF}$ coil 3 shown in Figure 10. With the values of the lumped elements at resonance frequency, it is charged by a step

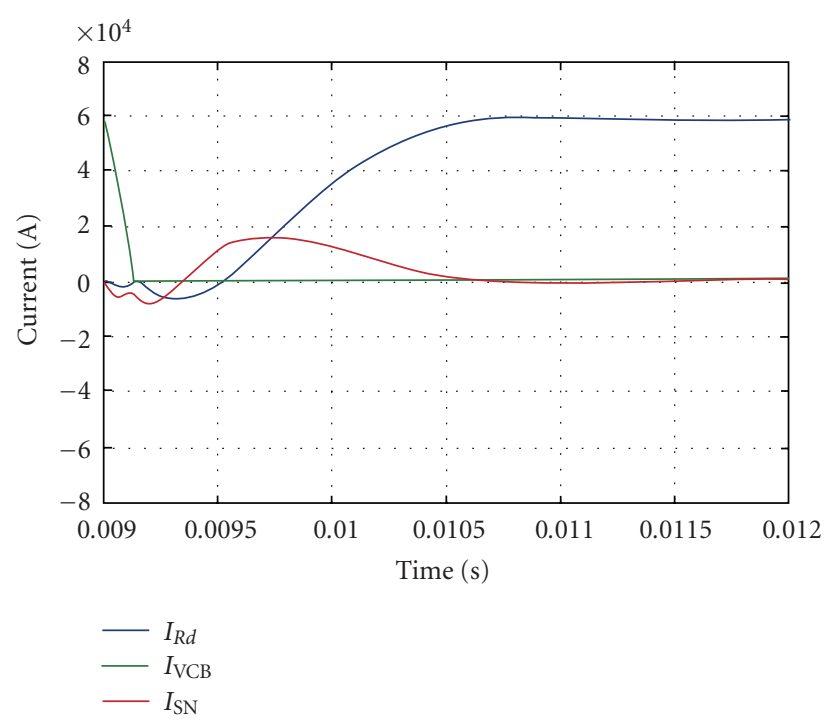

FIGURE 13: Results of TF fast discharge transient analysis; $I_{\mathrm{VCB}}$ : current through the VCB; $I_{R d}$ : current in the discharge resistor; $I_{\mathrm{SN}}$ : current in the snubber circuit.

function with the maximum occurring potential of $4.6 \mathrm{kV}$ in fault case. Then the power supply is interrupted and the rise time of the potential is determined to be 8 microseconds.

Now a step function with that rise time is applied to the detailed model. Three cases are considered after a voltage of $4.6 \mathrm{kV}$ is reached:

(i) voltage keeps constant (step function);

(ii) the power supply is switched off (fault case 1);

(iii) the power supply is switched off and an earth fault occurs (fault case 2). 


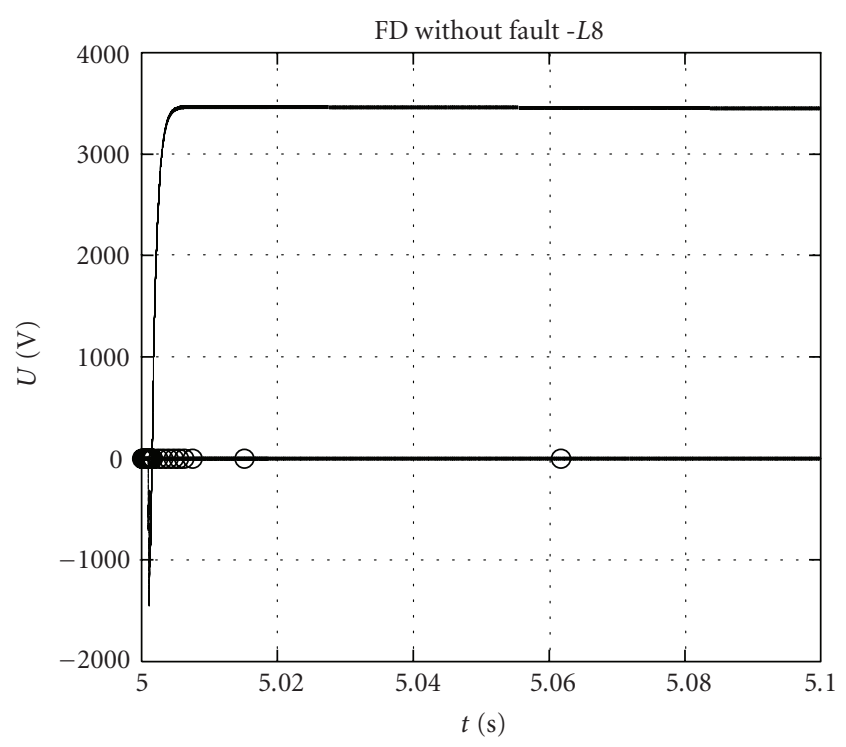

$\begin{array}{ll}- & U_{L 8 \text { terminal } 1} \\ - & U_{L 8 \text { terminal } 2}\end{array}$

Figure 14: Fast discharge without fault. Terminal voltage to ground (e.g., $L 8: 2), 3467 \mathrm{~V}(t=5.0085$ seconds), rise time from zero point to maximum: 1.6 milliseconds, capacitor discharge: $-696 \mathrm{~V}$, rise time: 1.1 microsecond ( $t=5.00100$ seconds), terminal to terminal voltage (e.g., $L 8): 3467 \mathrm{~V}$, rise time: 1.6 milliseconds $(t=5.0085$ seconds).

TABle 2: Overview of the calculated maximum voltage at ground, radial plate, and conductor insulation for the $1 \mathrm{kHz}$ and $50 \mathrm{kHz}$ model (the elements of network are calculated for $1 \mathrm{kHz}$ and $50 \mathrm{kHz}$, resp.) of TF coil.

\begin{tabular}{lcccc}
\hline & $f(\mathrm{kHz})$ & \multicolumn{3}{c}{ Maximum calculated voltage $(\mathrm{kV})$} \\
\hline $\begin{array}{l}\text { Insulation } \\
\text { type }\end{array}$ & & Fast discharge & $\begin{array}{l}\text { Fault } \\
\text { case 1 }\end{array}$ & $\begin{array}{l}\text { Fault } \\
\text { case 2 }\end{array}$ \\
\hline Ground & 1 & 3.5 & 8.1 & 16.4 \\
& 50 & 3.5 & 8.1 & 16.4 \\
Radial plate & 1 & 0.7 & 0.7 & 4.8 \\
& 50 & 0.6 & 0.7 & 4.8 \\
Conductors & 1 & 0.6 & 0.8 & 4.3 \\
& 50 & 0.5 & 0.8 & 3.7 \\
\hline
\end{tabular}

Because of the capacitances in the detailed model, there are oscillations in the coil which result in overvoltages. Results can be seen in Table 4.

For modelling the PF coils, the software ATP [12] was used. ATP does not allow including the coupling matrix of the complete PF system. So the influence of the other PF coils was not considered.

\section{Conclusion}

The numerical investigations of the transient behaviour of the ITER TF coils during fast discharge in normal case

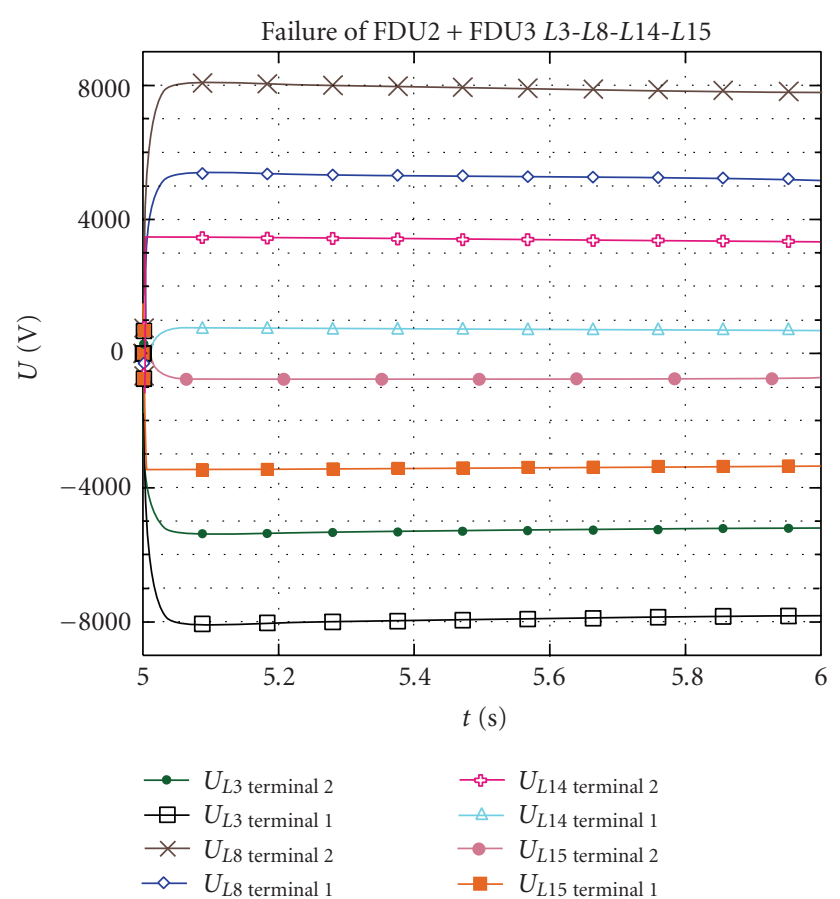

FIGURE 15: Voltages to ground on coil 3, 8, 14, and 15 during fast discharge with failure of FDU2 and FDU3.

TABLE 3: DC and AC (peak value) test voltages of TF coils for ground, radial plate, and conductor insulation based on the calculated voltages for the two different fault cases.

\begin{tabular}{lcc}
\hline & \multicolumn{2}{c}{ Test voltages $(\mathrm{kV})$} \\
\hline Insulation & Based on fault case 1 & Based on fault case 2 \\
\hline Ground & 18 & 34 \\
Radial plate & 5 & 11 \\
Conductor & 5 & 11 \\
\hline
\end{tabular}

TABle 4: Overview of the calculated maximum voltages for the $21.5 \mathrm{kHz}$ model of PF coil 4 .

\begin{tabular}{lccc}
\hline & \multicolumn{3}{c}{ Maximum calculated voltage $(\mathrm{kV})$} \\
\hline Insulation type & Step function & Fault case 1 & Fault case 2 \\
\hline Ground & 4.8 & 3.3 & 2.9 \\
Radial plate & 0.9 & 0.9 & 1.0 \\
Conductors & 1.0 & 0.9 & 1.2 \\
\hline
\end{tabular}

and in fault case show a significant increase of the radial plate and conductor insulation stress as a consequence of a malfunction of two adjacent FDUs followed by an additional earth fault. The design and test voltages must be chosen depending on the realistic fault scenarios identified by the ITER community.

For the poloidal field coils, the PF coil 4 shows the highest terminal potential during fast discharge with the fault of one FDU. As it displays overvoltages, the insulation coordination has to be selected. 


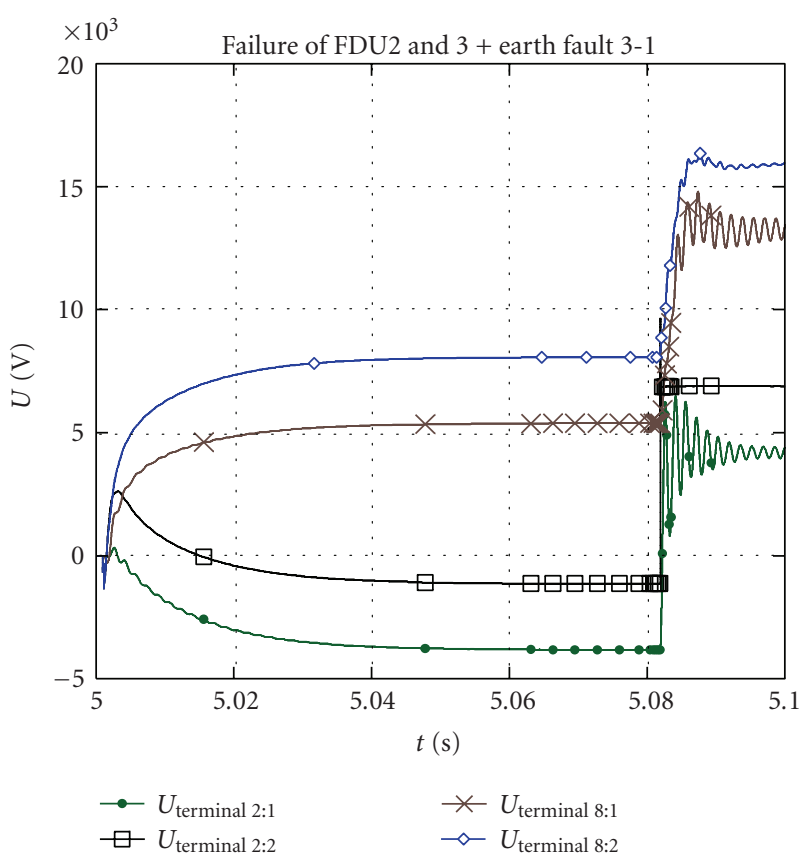

FIGURE 16: Fast discharge with failure of 2 FDUs and earth fault. Maximum voltage at terminal: $L 8: 2, U=16.35 \mathrm{kV}$ with oscillations $t=5.087$ seconds, rise time from $8.06 \mathrm{kV}$ to maximum voltage: 3 , 5 milliseconds, maximum terminal to terminal voltage: $L 2, U=$ $13.49 \mathrm{kV}$ at $t=5.0819$ seconds, rise time from $2.69 \mathrm{kV}$ to maximum $\mathrm{kV}: 2.4$ microseconds.

Further activities have to be done on central solenoid and its magnetic interactions with PF coils and the plasma under fault conditions during fast discharge.

\section{References}

[1] http://www.iter.org/.

[2] A. M. Miri, C. Meinecke, N. A. Riegel, A. Ulbricht, and S. Fink, "Influence of the radial plates on the transient oscillations in the ITER toroidal field (TF) model coil using a finite element approach," IEEE Transactions on Applied Superconductivity, vol. 10, no. 1, pp. 596-599, 2000.

[3] A. M. Miri and N. Riegel, "Transient behaviour of the ITER toroidal field (TF) model coil-finite element and network analysis," in Proceedings of the 7th International Conference on Optimisation of Electrical and Electronic Equipment (OPTIM '00), vol. 2, Brasov, Romania, May 2000.

[4] C. Meinecke, A. M. Miri, A. Ulbricht, and S. Fink, "Investigation of the transient electrical behavior of the ITER central solenoid model coil (CSMC) during safety discharge," IEEE Transactions on Applied Superconductivity, vol. 12, no. 1, pp. 1448-1452, 2002.

[5] S. Fink, A. Ulbricht, H. Fillunger, A. Bourquard, and M. Prevot, "High voltage tests of the ITER toroidal field model coil insulation system," IEEE Transactions on Applied Superconductivity, vol. 12, no. 1, pp. 554-557, 2002.

[6] A. Ulbricht, J. L. Duchateau, W. H. Fietz, et al., "The ITER toroidal field model coil project," Fusion Engineering and Design, vol. 73, no. 2-4, pp. 189-327, 2005.

[7] C. Meinecke, FE Modellierung der ITER TF Spule zur Untersuchung ihres transienten Verhaltens bei Schnellentladung unter Berücksichtigung des Skin- und Proximity-Effekts, IEH, Universität Karlsruhe, Karlsruhe, Germany, 1996.

[8] X. Quan, "Modellbildung eines supraleitenden Großmagneten zur Untersuchung seines transienten Verhaltens".

[9] DDD 11 Magnet 2.4: Fault and safety analyses.

[10] ITER Design Description Document DDD4.1 Figure 2.2.2-7, no. 2, 2001.

[11] N. Riegel, FEM- und Netzwerksimulationen des transienten Verhaltens großer supraleitender Spulen und ihrer metallischen Umgebung, Logos, Berlin, Germany, 1999.

[12] Alternative Transient Programm ATP Draw 3.8, p. 4. 

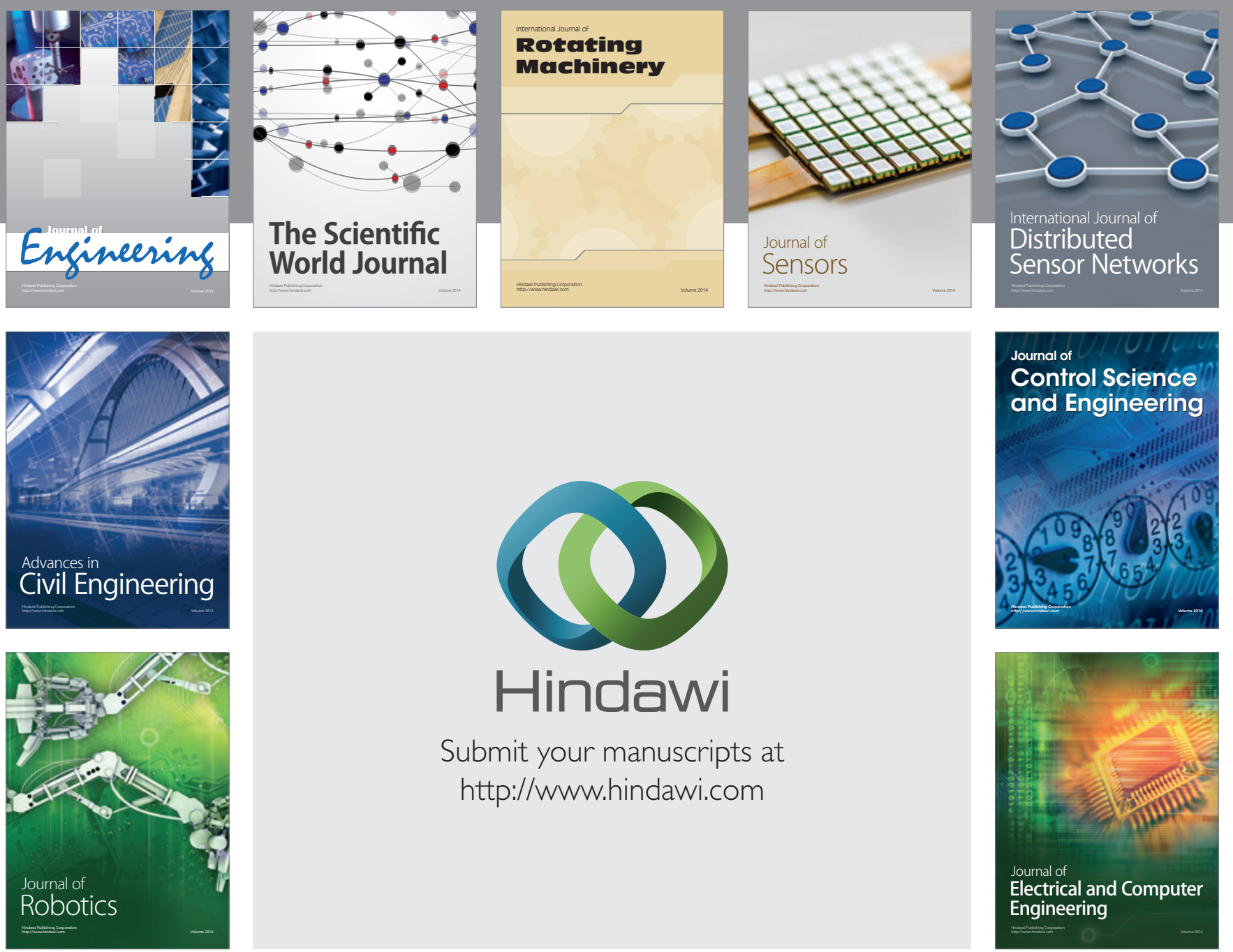

Submit your manuscripts at

http://www.hindawi.com
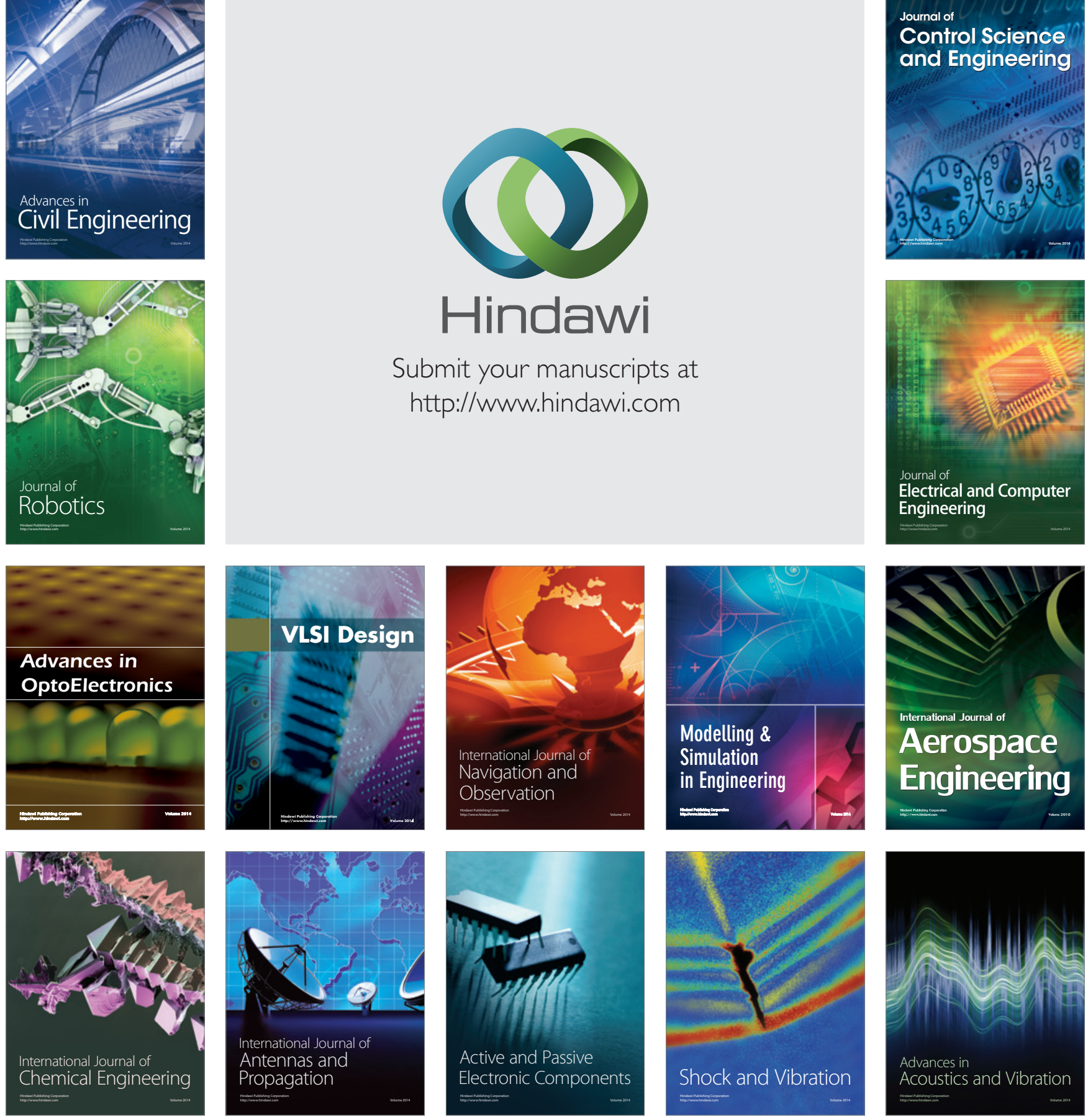